Research

Open Access

\title{
Acute and long-term survival in chronically critically ill surgical patients: a retrospective observational study
}

\author{
Wolfgang H Hartl ${ }^{1}$, Hilde Wolf ${ }^{1}$, Christian $\mathrm{P}$ Schneider ${ }^{1}$, Helmut Küchenhoff ${ }^{2}$ and Karl- \\ Walter Jauch1
}

\author{
1'Department of Surgery, Klinikum Grosshadern, Marchioninistr. 15, LMU Munich, D-81377 Munich, Germany \\ 2Institute of Statistics, Akademiestr. 1, LMU Munich, D-80799 Munich, Germany \\ Corresponding author: Wolfgang $\mathrm{H}$ Hartl, whartl@med.uni-muenchen.de \\ Received: 18 Dec 2006 Revisions requested: 31 Jan 2007 Revisions received: 3 Apr 2007 Accepted: 15 May 2007 Published: 15 May 2007 \\ Critical Care 2007, 11:R55 (doi:10.1186/cc5915) \\ This article is online at: http://ccforum.com/content/11/3/R55 \\ (c) 2007 Hartl et al.; licensee BioMed Central Ltd \\ This is an open access article distributed under the terms of the Creative Commons Attribution License http://creativecommons.org/licenses/by/2.0, \\ which permits unrestricted use, distribution, and reproduction in any medium, provided the original work is properly cited.
}

\begin{abstract}
Introduction Various cohort studies have shown that acute (short-term) mortality rates in unselected critically ill patients may have improved during the past 15 years. Whether these benefits also affect acute and long-term prognosis in chronically critically ill patients is unclear, as are determinants relevant to prognosis.
\end{abstract}

Methods We conducted a retrospective analysis of data collected from March 1993 to February 2005. A cohort of 390 consecutive surgical patients requiring intensive care therapy for more than 28 days was analyzed.

Results The intensive care unit (ICU) survival rate was $53.6 \%$. Survival rates at one, three and five years were $61.8 \%, 44.7 \%$ and $37.0 \%$ among ICU survivors. After adjustment for relevant covariates, acute and long-term survival rates did not differ significantly between 1993 to 1999 and 1999 to 2005 intervals. Acute prognosis was determined by disease severity during ICU stay and by primary diagnosis. However, only the latter was independently associated with long-term prognosis. Advanced age was an independent prognostic determinant of poor shortterm and long-term survival.

Conclusion Acute and long-term prognosis in chronically critically ill surgical patients has remained unchanged throughout the past 12 years. After successful surgical intervention and intensive care, long-term outcome is reasonably good and is mainly determined by age and underlying disease.

\section{Introduction}

Several studies have identified significant improvements in acute (short-term) mortality in the general intensive care unit (ICU) population throughout the past decade [1-10]. However, it is unclear whether advances in acute care can be translated into benefits in terms of long-term prognosis, and whether specific subgroups of critically ill patients may profit to a greater or lesser extent [11].

One possible way to define subgroups of critically ill patients is to classify them according to their length of stay in the intensive care unit (ICU). In the past, prolonged intensive care therapy (mostly related to need for mechanical ventilation) has variously been defined as more than 24 hours, more than 2 days, more than 14 days, or more than 28 days [12]. Unfortunately, the findings of studies examining ICU populations with variable length of stay cannot be compared because the degree of critical illness varies directly with length of ICU stay, and because the magnitude of the latter reflects a progressive selection process (survival of the fittest) [13].

Thus far, only five reported studies [14-18] have examined long-term prognosis in critically ill patients with a particularly long length of stay in the ICU ( $>28$ days). None of these studies examined variables relevant to long-term survival or survival time, although it is likely that, because of progress made in acute care, the number of patients now entering such a chronic state will rise.

Our aim in the present study was to analyze secular changes in acute and long-term mortality in patients who have undergone ICU therapy of duration in excess of 28 days, and to 
identify prognostic factors that are relevant to acute and longterm prognosis.

\section{Materials and methods Setting and population}

The analysis was conducted in the surgical ICU of the LudwigMaximilians University Hospital Klinikum Grosshadern in Munich, Germany, which is a 12-bed ICU that mostly receives postoperative patients from the Hospital. Staffing was exclusively surgical and included two senior, board-certified staff intensivists and nine residents (four to five of them were senior residents with at least one year of experience in surgical intensive care). A 12-hour shift system was used throughout the study, with at least one experienced physician in attendance at all times. The nurse/patient ratio varied between 1:2 and 1:3. ICU organization and management were identical during the period of study, meaning that ICU processes and admission, discharge, do-not-resuscitate order and withdrawal of care policies were consistent over time.

The inclusion period extended from 1 March 1993 to 28 February 2005 . The observation period started in 1993 , when an electronic chart was initiated in our ICU for local benchmarking and in-hospital information transfer. Survival status in all patients was obtained until 28 February 2007. A variety of new therapeutic strategies such as use of low tidal volumes or strict glycaemic control (for review [19]) were applied successively from 1999 onward.

We conducted a retrospective search of all eligible patients, including all consecutive patients admitted immediately or following a delay after a surgical procedure. Because of their small number, all patients who had not undergone surgery during their present hospital stay or who had been admitted only for medical reasons were excluded. Patients who had not consented to undergo prolonged intensive care were excluded from the analysis. Only patients with an ICU stay of longer than 28 days were included. The retrospective data analysis was approved by the local institutional review board. Baseline data and acute outcomes of the entire patient population treated in our institution between 1993 and 2005, and of a specific subpopulation (patients with an ICU length of stay $>4$ days) were recently reported $[1,20]$.

\section{Data collection}

We prospectively collected the following information for each patient: age; sex; admission and discharge dates from the ICU; outcome at ICU discharge; cause of death during ICU stay; primary diagnosis (abdominal disease, thoracic disease [mostly pulmonary malignancy], vascular disease, orthopaedic disease, combined diseases, severe sepsis as previously defined [21], pneumonia as previously defined [22], or peritonitis as previously defined [23]); admission state (emergency admission, readmission, immediate postoperative admission, surgery for a benign disease, curative surgery for a malignant disease, palliative surgery for a malignant disease); Acute Physiology and Chronic Health Evaluation (APACHE) II score during the first 24 hours after admission; maximum APACHE II score during ICU stay; maximal number of failing organs during ICU stay (organ failure was defined according to a modified Goris score [24]); and variables related to ICU therapy (duration of invasive mechanical ventilation, duration of catecholamine therapy, need for renal replacement therapy, number of transfused blood units) or to surgical therapy (number of reoperations).

Readmission was defined as an ICU admission after any preceding ICU admission that occurred during the same hospital stay and that lasted less than four weeks. Days or data from the preceding ICU admission were not used in the analysis, except that the patient's admission state was labelled as readmission. If a patient had already stayed on the ICU for more than four weeks, and if they could be discharged later but had to be readmitted a second time, then the patient was included in the study, but the second stay was ignored in the analysis. Sequential organ dysfunction and maximum organ dysfunction were monitored by daily calculation of APACHE II score, because specific methods (Sequential Organ Failure Assessment) were not yet available in 1993 [25].

\section{Statistical methods}

Regression modelling of mortality and time to death data Effects on acute prognosis were either evaluated by analyzing ICU mortality or time to death after inclusion. This duplicate analysis accounted for confounding effects arising from patient transfer to other ICUs or long-term care units. Furthermore, to identify factors that were exclusively relevant to longterm prognosis, we examined two-year mortality in ICU survivors.

Effects of variables were examined by logistic regression analysis and by nonproportional hazard models. Interactions between certain variables (APACHE II score on admission day, maximum APACHE II score during ICU stay, maximum number of failing organs during ICU stay) were also evaluated. The assumption that the effect was linear in the continuous variables was tested using the smoothed scatter plot approach proposed by Kay and Little [26] or by analyzing the effect of estimated coefficients of design variables (quartiles of the covariate distribution) on mortality or cumulative hazard rate [27]. In case of a nonlinear effect, a logarithmic, exponential, power, or quadratic transformation of the variable was tested. If these approaches failed to fit the data, then the covariate was divided into two classes based on median or quartiles [27].

Variables found to be associated with ICU mortality or twoyear mortality (ICU survivors) in the univariate analysis $(P<$ $0.20, P<0.01$ for interactions [28]) were entered into a stepwise multivariable logistic regression model to estimate 
adjusted odds ratios and 95\% confidence intervals. Statistical significance was defined as $P<0.05$. Goodness of fit was evaluated using Hosmer-Lemeshow statistics.

Effects of variables on survival time during the first two years after inclusion were initially examined using proportional hazard models. The form of relationship between two-year survival and patient variables, and the validity of the assumption of proportional hazards were investigated using plots based on Schoenfeld residuals [29]. These residuals revealed multiple violations of proportional hazards. Because nonproportional effects occurred in all variables before/after days 130 to 150 after inclusion, the time axis was partitioned by censoring all patients either still at risk at 150 days or who had already died before that time point [29]. Thereby, effects on five-month survival and on two-year survival in 150-day survivors could be analyzed separately. Also, within those two separate analyses we generated time-dependent covariates by creating interactions of the predictors and a logarithmic function of survival time, and included both in a combined model. If any of the time-dependent covariates were significant, then those predictors were considered not to be proportional.

Subsequently, a multivariate nonproportional hazard model with backward stepwise elimination of variables was constructed to estimate adjusted hazard ratios and 95\% confidence intervals. Variables with a $P$ value below 0.20 , timedependent covariates with a $P$ value below 0.10 , and interactions with a $P$ value below 0.01 by univariate analysis were entered into the model [28]. Statistical significance was defined as $P<0.05$.

Analysis of long term survival beyond the second year after inclusion

Kaplan-Meier survival analysis was used to describe long-term survival after the second year after inclusion (day 28 of intensive care therapy) and to compare survival rates with those of the German average population [30]. For the latter, an ideal reference population was constructed in which the members were all at the same age, matching the mean age of the comparison group.

Data presentation and between-group comparisons

Categorical variables were described as percentage and continuous variables as mean \pm standard deviation. A $P$ value of 0.05 or less in a two-tailed $\chi^{2}$ test was considered statistically significant.

\section{Power analysis}

One goal of the study was to evaluate differences in long-term survival between two successive six-year periods. A retrospective sample size calculation [29] indicated that 220 events (number of deaths) would allow detection of a $15 \%$ absolute increase in five-month survival rate (after inclusion) among critically ill patients with a presumed five-month survival rate of $40 \%$, at a significance level of $5 \%$ and a power of $90 \%$.

The statistical analysis was performed using a SAS Package (SAS version 9.1.3, 2002-2003; SAS Institute Inc., Cary, NC, USA) and an R package (version 2.4.1; R foundation, Vienna, Austria).

\section{Results \\ Clinical results}

During the 12-year period of observation, 392 patients had a stay in the ICU stayed of more than 28 days and fulfilled our criteria for inclusion in the cohort. Two patients $(0.05 \%)$ were lost to follow up and were excluded from the analysis. Clinical data for the whole cohort are presented in Table 1. Surgical ICU length of stay was $62.8 \pm 46.4$ days, surgical ICU survival rate was $53.6 \%$ and 150 -day survival rate after inclusion was $42.3 \%$. About half of the surgical ICU patients who had acutely survived their surgical disease were transferred to secondary ICUs in other institutions for weaning after long-term ventilatory support or for neurological/physical rehabilitation (Figure 1). The remaining surgical ICU survivors could be discharged to regular wards and were either directly transferred back to the referring hospital or remained at our institution. Almost half of the latter patients were later transferred to primary/secondary hospitals or rehabilitation centres, whereas most of the remaining patients could be discharged to home.

\section{Long-term survival rate}

Unadjusted long-term survival rates after inclusion, after surgical ICU discharge, or after day 150 or year 5 after inclusion are presented in Table 2 and in Figures 2 and 3 (Kaplan-Meier analyses). There were no significant differences between male and female patients. After surgical ICU discharge, survival rates were persistently lower than those of the German general population. Similar results were obtained when long-term survival was analyzed in patients who survived for longer than 150 days after inclusion (Figure 2). In the latter subgroup fiveyear survival after inclusion was $55.7 \%$ and 12 -year survival was $29.0 \%$. Long-term survival rates were clearly less than predicted, and even in patients surviving more than five years life expectancy was significantly shorter that in the German general population (Figure 3).

\section{Effect of admission date on outcome}

Because admission data could not be fitted by arithmetic transformations, these data were divided into two classes based on the median (before and after 1 March 1999). Crude ICU survival rates were comparable between the two time intervals $(51.5 \%$ during the period from 1993 to 1999 , and $54.7 \%$ during the period from 1999 to 2005; not significant). Correspondingly, there were no differences in long-term survival after inclusion. Unadjusted one-year, three-year and fiveyear survival rates after inclusion added up to 35.4\%, 25.9\% and $22.2 \%$ during the interval between 1993 and 1999 , and 
Critical Care Vol 11 No 3 Hartl et al.

Table 1

Baseline characteristics, clinical variables and variables of intensive care therapy

\begin{tabular}{|c|c|}
\hline Variable & Value \\
\hline Number of patients & 390 \\
\hline Age (years) & $65.3 \pm 13.5(67.0 ; 58.0-75.0)$ \\
\hline Sex (\% male) & 71.5 \\
\hline Emergency admission (\%) & 60.4 \\
\hline Readmission (\%) & 12.3 \\
\hline Immediate postoperative admission (\%) & 66.2 \\
\hline \multicolumn{2}{|l|}{ Surgical speciality (\%) } \\
\hline Abdominal surgery & 48.8 \\
\hline Thoracic surgery & 17.7 \\
\hline Vascular surgery & 20.8 \\
\hline Orthopaedic surgery & 9.8 \\
\hline Combined surgery $(\%)$ & 1.5 \\
\hline Benign disease (\%) & 66.3 \\
\hline Curative surgery for malignant disease (\%) & 21.9 \\
\hline Palliative surgery for malignant disease (\%) & 11.8 \\
\hline APACHE II score on admission day & $18.4 \pm 6.9(18.0 ; 13.0-23.3)$ \\
\hline Pneumonia (\%) & 68.1 \\
\hline Peritonitis (\%) & 30.8 \\
\hline Severe sepsis (\%) & 61.1 \\
\hline Need for mechanical ventilation (\%) & 99.0 \\
\hline Duration of mechanical ventilation (days) & $44.8 \pm 44.7(31.0 ; 17.0-57.3)$ \\
\hline Need for catecholamine therapy (\%) & 92.3 \\
\hline Duration of catecholamine therapy (days) & $28.3 \pm 30.4(18.0 ; 6.0-32.0)$ \\
\hline Need for renal replacement therapy (\%) & 35.1 \\
\hline Duration of continuous renal replacement therapy (days) & $9.8 \pm 23.9(0.0 ; 0.0-7.8)$ \\
\hline Need for red cell transfusion (\%) & 97.2 \\
\hline Number of transfused red blood cell units & $21.8 \pm 26.0(14.0 ; 6.0-28.0)$ \\
\hline Number of surgical revisions & $2.1 \pm 3.0(1.0 ; 0.0-3.0)$ \\
\hline Maximum APACHE II score during ICU stay & $29.4 \pm 6.9(30 ; 25.0-34.0)$ \\
\hline Maximum number of failing organs & $4.4 \pm 1.4(5 ; 3-6)$ \\
\hline
\end{tabular}

Continuous data are presented as mean \pm standard deviation (median; $25 \%$ to $75 \%$ quartile). APACHE, Acute Physiology and Chronic Health Evaluation; ICU, intensive care unit.

to $30.5 \%, 21.6 \%$ and $13.7 \%$ during the interval between 1999 and 2005 (not significant, according to log-rank testing). Also, after adjusting for potential confounders, acute and twoyear prognosis was not affected significantly by admission date (before or after 1 March 1999; Table 3). However, we observed a significant difference with respect to cause of death during surgical ICU stay. Single organ failure as the cause of death was significantly more common in patients dying before March 1999 (23.0\%) than in those dying thereafter $(9.9 \% ; P<0.05)$.

\section{Determinants of acute prognosis}

Multivariate analysis identified advanced age, duration of catecholamine therapy, surgery for thoracic diseases, peritonitis, maximum APACHE II score during the surgical ICU stay, and maximum number of failing organs as independently associated with ICU mortality (Table 4). The $P$ value from Hosmer- 
Figure 1

\section{Patient flow chart}

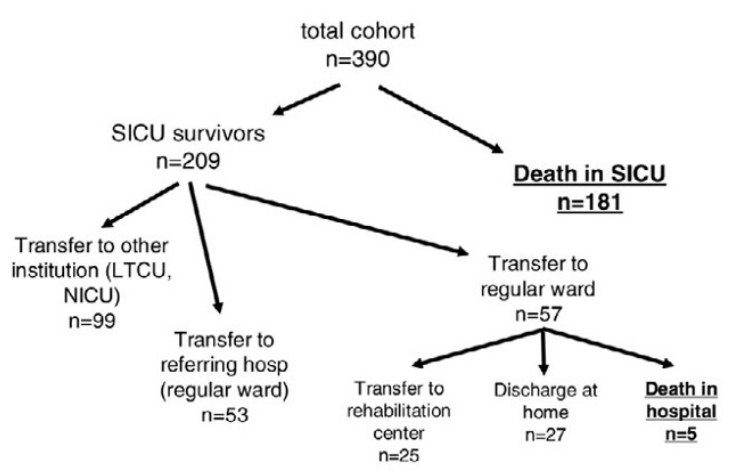

Patient flow after inclusion in the study. LTCU, long-term care unit; NICU, neurological intensive care unit; SICU, surgical intensive care unit.

Lemeshow statistical analysis was 0.935 . With the exception of duration of catecholamine therapy, the same variables could be identified as independent risk factors for time to death until day 150 after inclusion (Table 5). Additional determinants were pneumonia and the number of surgical revisions. The latter variable had a complex independent association with survival time, with only low number of surgical revisions being associated with prolonged survival time (Figure 4).

\section{Determinants of two-year prognosis}

Variables that were independent determinants of two-year mortality in ICU survivors were advanced age, surgery for thoracic disease and palliative surgery for malignant disease (Table 6). $P$ value from Hosmer-Lemeshow statistical analysis was 0.944 . Advanced age and surgery for thoracic disease were also independent risk factors for a shorter survival time in patients surviving more than 150 days, as were surgery for malignant diseases, duration of mechanical ventilation $(>50$ days), and the number of surgical revisions (Table 7). Again, a complex interaction of the latter variable with survival time was found, in which a lower number of surgical revisions was associated with a shorter survival time (Figure 5).

\section{Discussion \\ Magnitude of short-term and long-term survival}

Our analysis is the largest to describe the determinants and secular trends in acute and long-term mortality over a 12-year period in postoperative patients with an ICU length of stay of more than 28 days. We found that short-term prognosis in this particular patient group is limited (ICU survival rate $53.6 \%$, 150-day survival rate after inclusion 42.3\%). However, after successful surgery and intensive care therapy, long-term outcome in survivors is reasonably good, with five-year survival rates varying between 37\% (in ICU survivors) and 56\% (in patients surviving more than 150 days).

Acute survival rates in our mixed surgical cohort correspond well with those found by others in abdominal surgical, cardiac surgical, or mixed surgical/medical patients with a similar length of ICU stay [14,16,18,31]. Acute prognosis after chronic critical illness was only different among patients who were clearly younger $[17,32,33]$ or older [15] than ours. Also one-year survival rate in our ICU survivors was almost similar to that found by other investigators in patients at a similar age $[14,16]$ and was superior to that in older patients [15]. However, three-year and five-year survival rates in our cohort were about $10 \%$ lower than those seen after exclusively abdominal surgery [16] or in predominantly medical ICU patients [14] with a prolonged ICU length of stay and of similar age. The most likely explanation for this difference resides in the greater percentage of patients in our cohort who were suffering from malignant pulmonary diseases or had undergone palliative surgery. Both conditions may be expected to be associated with a less favourable long-term prognosis.

Compared with the general population, long-term survival

\section{Table 2}

Long-term survival after more than 28 days of intensive care therapy or after ICU discharge and in age-matched German general population

\begin{tabular}{|c|c|c|c|c|}
\hline \multirow[t]{2}{*}{ Time of assessment } & \multicolumn{4}{|c|}{ Survival } \\
\hline & 1 year & 2 years & 3 years & 5 years \\
\hline After day 28 & $33.0 \%$ & $27.0 \%$ & $23.9 \%$ & $19.8 \%$ \\
\hline After ICU discharge (male and female) & $61.8 \%$ & $50.6 \%$ & $44.7 \%$ & $37.0 \%$ \\
\hline After ICU discharge (male) & $64.7 \%$ & $53.8 \%$ & $46.0 \%$ & $37.7 \%$ \\
\hline After ICU discharge (female) & $53.8 \%$ & $41.6 \%$ & $41.6 \%$ & $36.3 \%$ \\
\hline General population (male, age 65 years) ${ }^{a}$ & $98.3 \%$ & $96.5 \%$ & $94.6 \%$ & $90.3 \%$ \\
\hline General population (female, age 65 years) ${ }^{a}$ & $99.2 \%$ & $98.4 \%$ & $97.4 \%$ & $95.2 \%$ \\
\hline
\end{tabular}

aData from Statistisches Bundesamt Wiesbaden, Germany [30]. 
Figure 2

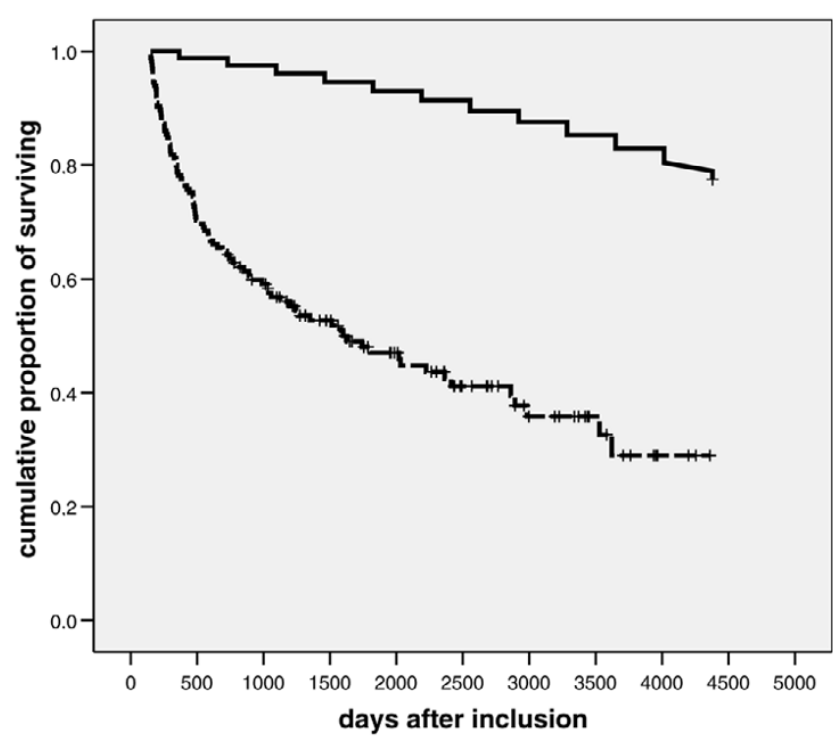

Twelve-year survival: chronically critically ill patients who have already survived 150 days versus general population. Presented are KaplanMeier plots showing 12-year survival rates (after inclusion) in patients surviving more than 150 days (dashed line) and in the German general population (continuous line; reference age 61 years; data from Statistisches Bundesamt Wiesbaden, Germany [30]).

rates after successful initial therapy were consistently lower in our patients, even beyond the fifth year. It is commonly believed that it may take 4 years or more for survival of ICU patients to parallel that in the general population [13]. However, this finding may only be valid for ICU populations with minor pre-existing illnesses. It is likely that independent effects of the primary disease process will be more important to long-term prognosis in surgical patients, who intrinsically suffer from major illnesses before the surgery. These diseases are the reason for the surgical intervention, and may only be superimposed temporarily by subsequent organ malfunction and consequent intensive care therapy.

\section{Prognostic factors}

The extent to which the consequences of prolonged critical illness or treatments received in the ICU contribute to mortality, and whether these are potentially reversible, is still poorly understood. An expert panel convened by the European Intensive Care Society, the American Thoracic Society, and the Society of Critical Care Medicine [13] has identified late deaths after critical illness as a priority research area. The lack of long-term data compares unfavourably with what is known about the long-term course of other disease groups such as heart disease and cancer [13,34-36]. Therefore, one aim of our study was to identify prognostic factors that determine survival in patients with prolonged ICU stay.

Our analysis is the first to allow quantification of independent effects of the primary disease, severity of illness during ICU
Figure 3

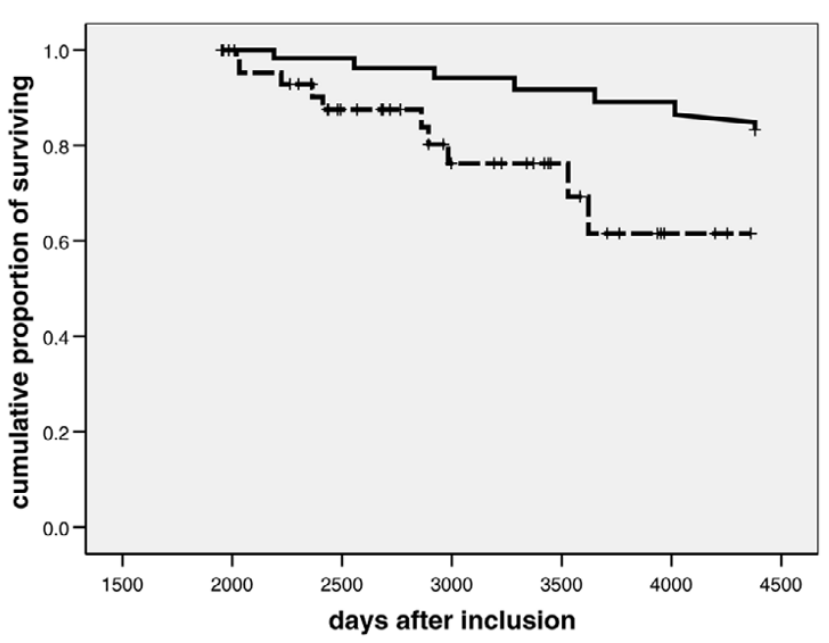

Twelve-year survival: patients who have already survived longer than five years versus general population. Presented are Kaplan-Meier plots showing 12-year survival rates (after inclusion) in patients having already survived for more than five years (dashed line) and in the German general population (continuous line; data from Statistisches Bundesamt Wiesbaden, Germany [30]). $P<0.001$ versus reference population of 1,000 individuals

stay and treatments applied during intensive care. According to our findings, the greater case fatality rate in long-term survivors must predominantly be attributed to pre-existing diseases, especially malignancies, the presence of which was a strong determinant of long-term survival. Our analysis also shows that variables that relate to disease severity during the ICU stay or to ICU therapy have a rather important influence on acute survival (Tables 4 and 5), but they are of almost no importance to long-term survival (Tables 6 and 7). The validity of these findings is supported by the fact that almost identical results were obtained by two different statistical methods (logistic regression analysis and nonproportional hazard analysis of survival time).

Several important conclusions may be drawn from our multivariate analysis, and these are discussed below.

\section{Age}

Old age represents a strong independent risk factor for both poor acute and poor long-term prognosis after prolonged critical care. This nonlinear effect of age on patient prognosis is suggested by the fact that only a quadratic or power transformation of the age data yielded the necessary linear association between age and outcome in three of the four statistical models used (Tables 4, 5, 6). However, in long-term survivors $(>150$ days after inclusion) the effect of age appeared to decrease over time, because the hazard ratio of the time-dependent covariate was under 1 (Table 7).

As was recently reviewed [13], age presumably influences long-term prognosis in critically ill patients to a large extent by 
Table 3

Covariate-adjusted effect of admission date (before versus after 1 March 1999) on acute and long-term prognosis

\begin{tabular}{lll}
\hline Prognosis & HR)/OR $(95 \% \mathrm{Cl})$ & $P$ value \\
\hline $\begin{array}{l}\text { Survival time until day } 150 \text { after inclusion } \\
\begin{array}{l}\text { Survival time until year } 3 \text { after inclusion } \\
\text { in patients surviving }>150 \text { days }\end{array}\end{array}$ & HR $1.206(0.871-1.670)$ & 0.260 \\
ICU mortality & HR $1.278(0.653-2.500)$ & 0.474 \\
Two-year mortality rate in ICU survivors & OR $1.169(0.551-2.481)$ & 0.684 \\
\hline
\end{tabular}

$\mathrm{Cl}$, confidence interval; $\mathrm{HR}$, hazard ratio; ICU, intensive care unit; OR, odds ratio.

being a marker for residual functional disability. However, it should be noted that, because of the nature of our study, patients with extreme physical disabilities were not included in our analysis. Because all of the patients included in the study had undergone elective or emergency surgery, their preoperative physical state must have been such that they were expected to survive at least the surgical procedure and the immediate postoperative phase.

\section{Duration of mechanical ventilation}

A particularly long duration of mechanical ventilation $(>50$ days) was the only independent variable that was related to ICU therapy and was found to be associated with shorter longterm survival in patients surviving longer than 150 days. A worse long-term prognosis after prolonged invasive ventilation ( $>49$ days) was previously suggested by the univariate analysis conducted by Gracey and coworkers [14]. This interaction was elaborated by subsequent studies that adjusted for potential confounders when evaluating patients who needed invasive ventilation for longer than 21 or 35 days $[37,38]$.

\section{Effectiveness/efficacy of surgery}

From the surgical perspective, there appears to be a fairly complex but significant association between surgical efficacy (as indicated by the number of surgical revisions) and outcome. Although the number of revisions was not associated with a significantly worse ICU or two-year survival by logistic regression analysis, it was a strong determinant of acute and long-term survival time (Figures 4 and 5 ). Thus, small numbers of surgical revisions lead to a longer survival time during the first months after inclusion but shortened survival time in patients surviving for longer than 150 days. On the other hand, a large number of surgical revisions (more than three or four) was not associated with a particularly poor or favourable acute or long-term prognosis. These findings may reflect a selection process in which a large number of re-operations is only possible in patients who are fit enough to withstand prolonged critical illness. Furthermore, these revisions will be only done in those patients judged likely to derive benefit from repeated interventions. However, we cannot completely exclude the possibility that those opposing effects on survival time were simply due to statistical heterogeneity and insufficient numbers of patients with multiple surgical revisions.

\section{Initial severity of illness}

APACHE II score in the first 24 hours after admission had no impact on acute or long-term prognosis in our patient cohort. The absence of an association between 24-hour APACHE II score and acute outcome after prolonged critical care was previously demonstrated [18,32]. The lack of influence of disease severity at admission on prognosis may once again suggest a selection process. Specifically, patients might not have not survived until week five either because they were too sick to respond to therapy or because they were among the ones who would have responded to therapy but did not receive

\section{Table 4}

\begin{tabular}{lll}
\multicolumn{2}{l}{ Independent risk factors for ICU mortality } & \\
\hline & Odds ratio (95\% confidence interval) & $P$ value \\
\hline Age (per year)a & $26.730(1.970-362.622)$ & 0.014 \\
Maximum APACHE II score (per point) & $1.567(1.200-2.047)$ & 0.001 \\
Duration of catecholamine therapy (per day) ${ }^{\mathrm{b}}$ & $10.188(2.789-37.215)$ & $<0.001$ \\
Maximum number of failing organs (per organ) & $6.913(1.356-35.244)$ & 0.020 \\
Surgery for thoracic diseases & $3.651(1.541-8.647)$ & 0.003 \\
Peritonitis & $6.437(3.068-13.505)$ & $<0.001$
\end{tabular}

aAfter quadratic transformation. ${ }^{b}$ After logarithmic transformation. APACHE, Acute Physiology and Chronic Health Evaluation; ICU, intensive care unit. 
Table 5

Survival time analysis until day $\mathbf{1 5 0}$ after inclusion (independent risk factors)

\begin{tabular}{|c|c|c|}
\hline & $\begin{array}{l}\text { Hazard ratio (95\% confidence } \\
\text { interval) }\end{array}$ & $P$ value \\
\hline Age (per year)a & $3.213(1.823-5.665)$ & $<0.001$ \\
\hline Maximum APACHE II score (per point)b & $15.311(5.860-40.005)$ & $<0.001$ \\
\hline Number of surgical revisions (per revision)c & $1.381(1.154-1.652)$ & $<0.001$ \\
\hline Time-dependent covariate for number of surgical revisions & $1.689(1.189-2.400)$ & 0.003 \\
\hline Maximum number of failing organs (per organ)c & $1.664(1.260-2.198)$ & $<0.001$ \\
\hline Pneumonia & $2.263(1.225-4.180)$ & 0.009 \\
\hline Time-dependent covariate for pneumonia & $1.480(1.121-1.954)$ & 0.006 \\
\hline Surgery for thoracic diseases & $1.975(1.335-2.921)$ & 0.001 \\
\hline Peritonitis & $1.789(1.278-2.504)$ & 0.001 \\
\hline
\end{tabular}

aAfter power transformation. ${ }^{b}$ After logarithmic transformation. ${ }^{A}$ After quadratic transformation. APACHE, Acute Physiology and Chronic Health Evaluation.

appropriate treatment. Furthermore, patients with minor disease severity will already have left the surgical ICU by that time. Therefore, disease severity during the ICU stay appears to be much more important for acute prognosis than the initial extent of organ dysfunction.

\section{Catecholamine therapy}

Duration of catecholamine therapy was an independent prognostic determinant only when ICU survival was analyzed. This acute effect corresponds to observations by others who also evaluated determinants of acute outcome in patients undergo-

Figure 4

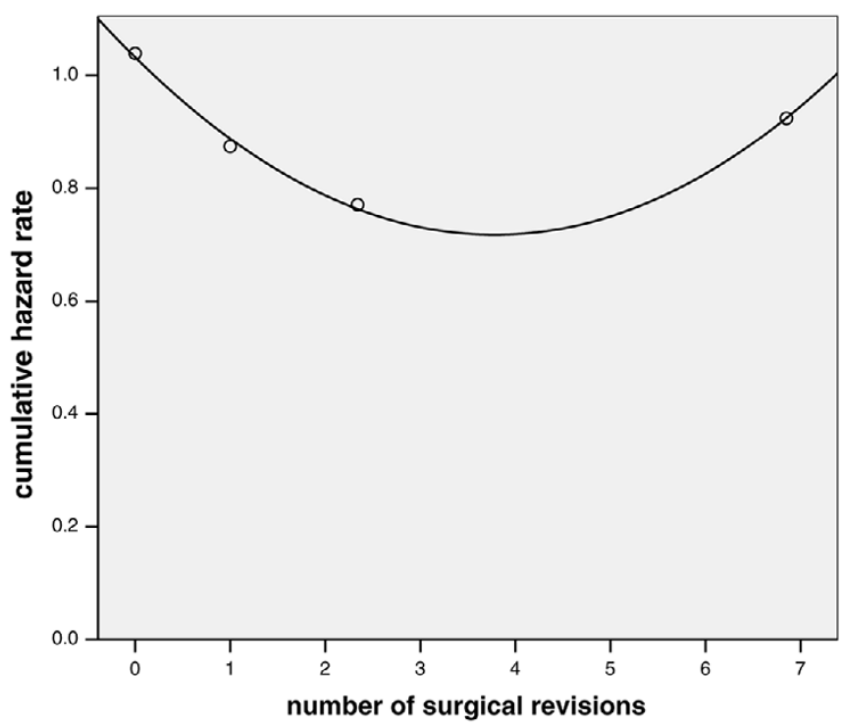

Univariate analysis of surgical efficacy versus cumulative hazard rate: first 150 days after inclusion. Shown is the univariate association between the number of surgical revisions (mean value per quartile) and the corresponding cumulative hazard rate for the first 150 days after inclusion. $P<0.001$ after quadratic transformation of continuous data, and addition of a time-dependent covariate. ing a very long stay in the ICU [18]. These findings emphasize the importance of ongoing circulatory failure (as reflected by the use of vasoactive drugs) to acute prognosis in prolonged critical illness.

\section{Malignancy}

In contrast to long-term prognosis, acute prognosis was not worsened by extended tumor disease. The lack of importance of tumour extent to acute survival has previously been demonstrated by several investigators and has stimulated the concept of conducting intensive care regardless of tumour stage $[39,40]$. It appears that even a prolonged ICU length of stay would not conflict with the application of such a concept during care in palliative patients.

\section{Secular changes}

A further aim of our study was to examine whether implementation of recent advances in critical care medicine has improved prognosis in chronically critically ill patients in our institution. We found that acute and long-term outcome had remained unchanged between 1993 and 2005 in our patients. The only significant secular change concerned the importance of single organ failure, which was less often a cause of death after 1999. Thus, it appears that treatment of individual organ failure (for instance, therapy for pulmonary failure) became more effective during the period of observation than did therapy for multiple organ failure. However, improved control of severe single organ failure might have allowed more patients to develop multiple organ dysfunction in later years. Multiple organ failure represents a highly complex condition in which therapeutic targets may often conflict with each other, thereby possibly preventing secular improvement in survival. The lack of improvement in acute prognosis is at odds with the findings of a variety of other studies [1-10], but it presumably emphasizes the extraordinary circumstances that may be encountered in patients with prolonged critical illness. It should be 
Table 6

Independent risk factors for two-year mortality in ICU survivors

\begin{tabular}{lll}
\hline & Odds ratio (95\% confidence interval) & $P$ value \\
\hline Age $\left(\right.$ per year) $^{\mathrm{a}}$ & $25.524(1.495-435.670)$ & 0.025 \\
Surgery for thoracic diseases & $3.004(1.223-7.379)$ & 0.016 \\
Palliative surgery & $23.863(3.098-183.788)$ & 0.002 \\
\hline
\end{tabular}

aAfter quadratic transformation. ICU, intensive care unit.

noted that our analysis only allows recognition of a relative improvement in short-term survival rate by about 15\% (absolute improvement in 150-day survival from $40 \%$ to $55 \%$ ). Therefore, we cannot exclude minor advances in prognosis. Two hypotheses may be proposed to account for the unchanged prognosis in surgical patients following prolonged critical illness.

First, recent evidence-based recommendations for intensive care therapy (such as strict glycaemic control or use of low tidal volumes during mechanical ventilation) have been derived from studies of interventions designed to treat an acute lifethreatening insult [19]. Patients who survive this initial intensive care period and remain in the ICU for prolonged periods of time (such as our cohort) may experience a second threat, which is likely to be related to the risks associated with the prolonged ICU stay and includes ventilator-acquired pneumonia, catheter or urinary tract infection, persistent abdominal septic foci, or multiple organ dysfunction. These secondary, recurrent threats may be much less susceptible to strategies developed to manage the initial insult and may ultimately kill the patient [41].

Second, it is possible that the acute survival benefit of evidence-based therapeutic strategies does not persist beyond hospital discharge. For example, analysis of the effect of drotrecogin alfa (activated) on long-term survival after severe sepsis demonstrated that treated patients had a higher survival rate at hospital discharge. However, there was no statistical difference between treatment arms in duration of survival or differences in survival rates at 3 months, 1 year and 2.5 years after discharge [11].

\section{Limitations of the study}

The present study has a number of limitations. Besides the primary diagnosis, a key role for ICU outcome determination must be attributed to specific structures or process qualities. More than 20 variables, such as length of shifts for house officers and nurse/patient ratio, have been identified as independent determinants of patient outcome in the ICU [42]. Although during the 12-year study period structures or processes not directly related to specific technical aspects of therapy remained largely unchanged on our ICU, we cannot completely exclude an effect of these potential confounders on the results of our study.

A further bias relevant to investigations of patient mortality may arise from the individual preferences of the treating physicians to continue or withdraw life support after a certain duration of ICU therapy [43]. Although the same senior intensivists were in charge during the entire period of study, a constant albeit subjective attitude toward discontinuation of life supportive measures cannot always be guaranteed.

In addition, the results of our study may not be generalizable because they represent the experience of a single centre and reflect a unique organization and process of care. Because there were no medical ICU patients or patients, for instance after cardiac surgery or neurosurgery, our findings may not be entirely applicable to patient cohorts others than ours.

Table 7

Survival time analysis until the third year after inclusion (independent risk factors) in patients surviving more than 150 days

\begin{tabular}{lll} 
& $P$ value & Hazard ratio (95\% confidence interval) \\
\hline Age (per year) & 0.019 & $1.044(1.007-1.083)$ \\
Time-dependent covariate for age & 0.028 & $0.949(0.905-0.994)$ \\
Duration of mechanical ventilation & 0.007 & $2.306(1.250-4.254)$ \\
Palliative surgery & $<0.001$ & $4.458(2.032-9.778)$ \\
Number of surgical revisions (per revision) & 0.005 & $0.097(0.019-0.495)$ \\
Surgery for malignant diseases & 0.010 & $2.339(1.227-4.460)$ \\
\hline
\end{tabular}

aFor patients ventilated for more than 50 days. ${ }^{b}$ After quadratic transformation. 
Figure 5

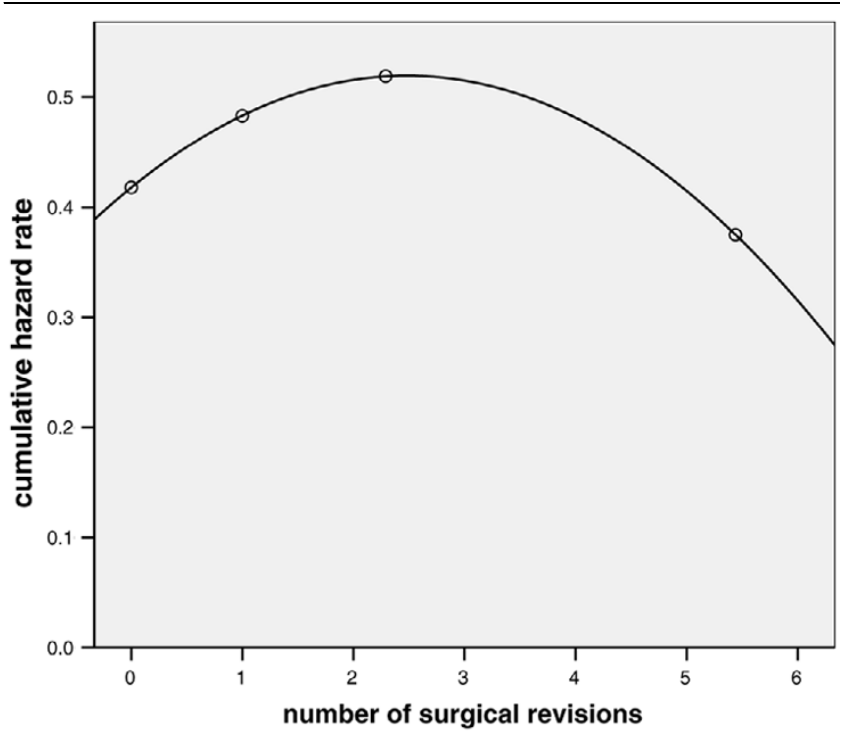

Univariate analysis of surgical efficacy versus cumulative hazard rate: first two years after inclusion. Univariate association between the number of surgical revisions (mean value per quartile) and the corresponding cumulative hazard rate for the first two years after inclusion in patients surviving more than 150 days. $P=0.033$ after quadratic transformation of continuous data.

On the other hand, our crude findings regarding acute and one-year survival rates corresponded well with findings in other patient cohorts with a comparable ICU length of stay or age, but with different primary diagnosis [14,16,18,31]. Therefore, we feel that at least some conclusions of our study may also valid for unselected populations of ICU patients. Such general conclusions may especially pertain to categories of determinants that influence acute and long-term prognosis.

\section{Conclusion}

Despite a high acute fatality rate, long-term prognosis in chronically critically ill surgical patients is reasonably good. However, it is not comparable to that of the general German population, even beyond the fifth year after inclusion. Acute survival is determined by disease severity during ICU stay and by pre-existing illnesses, whereas long-term survival mostly depends on the underlying disease. Older patients appear to be at a particularly high risk for death and shorter survival. Acute and long-term prognosis have not changed during the past 12 years.

\section{Competing interests}

The authors declare that they have no competing interests.

\section{Authors' contributions}

WH designed the study and drafted the manuscript. CPS and $\mathrm{HW}$ participated in generating data. HK participated in the design of the study and performed the statistical analysis. KWJ conceived the study, participated in its design and

\section{Key messages}

- No change in acute and long-term survival of chronically critically ill surgical patients has occurred at our institution over the past decade.

- Disease severity during ICU stay is a strong determinant of acute prognosis, but it is of almost no importance to long-term prognosis, which is mainly determined by preexisting illnesses.

- Long-term survival in chronically critically ill surgical patients is reasonably good after successful surgery and intensive care therapy, but it is not comparable to that in the general population.

coordination, and helped to draft the manuscript. All authors read and approved the final manuscript.

\section{Acknowledgements}

The authors thank D Inthorn and H Schneeberger for initiation and maintenance of the database.

\section{References}

1. Hartl WH, Wolf H, Schneider CP, Küchenhoff H, Jauch KW: Secular trends in mortality associated with new therapeutic strategies in surgical critical illness. Am J Surg 2007 in press.

2. Annane D, Aegerter P, Jars-Guincestre MC, Guidet B, CUB-Rea Network: Current epidemiology of septic shock: the CUB-Rea Network. Am J Respir Crit Care Med 2003, 168:165-172.

3. Martin GS, Mannino DM, Eaton S, Moss M: The epidemiology of sepsis in the United States from 1979 through 2000. N Engl J Med 2003, 348:1546-1554.

4. Dombrovskiy VY, Martin AA, Sunderram J, Paz HL: Facing the challenge: decreasing case fatality rates in severe sepsis despite increasing hospitalizations. Crit Care Med 2005 , 33:2555-2562.

5. Stapleton RD, Wang BM, Hudson LD, Rubenfeld GD, Caldwell ES, Steinberg KP: Causes and timing of death in patients with ARDS. Chest 2005, 128:525-532.

6. Suchyta MR, Orme JF Jr, Morris AH: The changing face of organ failure in ARDS. Chest 2003, 124:1871-1879.

7. Azoulay E, Alberti C, Bornstain C, Leleu G, Moreau D, Recher C, Chevret S, Le Gall JR, Brochard L, Schlemmer B: Improved survival incancer patients requiring mechanical ventilatory support: impact ofnoninvasive mechanical ventilatory support. Crit Care Med 2001, 29:519-525.

8. Larche J, Azoulay E, Fieux F, Mesnard L, Moreau D, Thiery G, Darmon M, Le Gall JR, Schlemmer B: Improved survival of critically ill cancer patients with septic shock. Intensive Care Med 2003 29:1688-1695.

9. Moran JL, Solomon PJ, Williams PJ: Assessment of outcome over a 10-year period of patients admitted to a multidisciplinary adult intensive care unit with haematological and solid tumours. Anaesth Intensive Care 2005, 33:26-35.

10. Girou E, Brun-Buisson C, Taille S, Lemaire F, Brochard L: Secular trends in nosocomial infections and mortality associated with noninvasive ventilation in patients with exacerbation of COPD and pulmonary edema. JAMA 2003, 290:2985-2991.

11. Angus DC, Laterre PF, Helterbrand J, Ely EW, Ball DE, Garg R, Weissfeld LA, Bernard GR, PROWESS Investigators: The effect of drotrecogin alfa (activated) on long-term survival after severe sepsis. Crit Care Med 2004, 32:2199-2206.

12. Maclntyre NR, Epstein SK, Carson S, Scheinhorn D, Christopher $\mathrm{K}$, Muldoon S: Management of patients requiring prolonged mechanical ventilation: report of a NAMDRC consensus conference. Chest 2005, 128:3937-3954.

13. Williams TA, Dobb GJ, Finn JC, Webb SA: Long-term survival from intensive care: a review. Intensive Care Med 2005 31:1306-1315. 
14. Gracey DR, Naessens JM, Krishan I, Marsh HM: Hospital and posthospital survival in patients mechanically ventilated for more than 29 days. Chest 1992, 101:211-214.

15. Montuclard L, Garrouste-Orgeas M, Timsit JF, Misset B, De Jonghe $\mathrm{B}$, Carlet J: Outcome, functional autonomy, and quality of life of elderly patients with a long-term intensive care unit stay. Crit Care Med 2000, 28:3389-3395.

16. Neipp M, Jähne J, Niechzial M, Pichlmayr R: Prolonged intensive care stay after abdominal surgery interventions with special reference to quality of life, occupational rehabilitation and economics. Chirurg 1997, 68:410-415.

17. Goins WA, Reynolds HN, Nyanjom D, Dunham CM: Outcome following prolonged intensive care unit stay in multiple trauma patients. Crit Care Med 1991, 19:339-345.

18. Friedrich JO, Wilson G, Chant C: Long-term outcomes and clinical predictors of hospital mortality in very long stay intensive care unit patients: a cohort study. Crit Care 2006, 10:R59.

19. Dellinger RP, Carlet JM, Masur H, Gerlach H, Calandra T, Cohen J, Gea-Banacloche J, Keh D, Marshall JC, Parker MM, et al.: Surviving Sepsis Campaign guidelines for management of severe sepsis and septic shock. Crit Care Med 2004, 32:858-873.

20. Schneider CP, Wolf $H$, Küchenhoff $H$, Jauch $K W$, Hartl WH: Trends in surgical intensive care. Experience in one centre over 12 years [in German]. Chirurg 2006, 77:700-708.

21. Bone RC, Balk RA, Cerra FB, Dellinger RP, Fein AM, Knaus WA, Schein RM, Sibbald WJ: Definitions for sepsis and organ failure and guidelines for the use of innovative therapies in sepsis. The ACCP/SCCM Consensus Conference Committee. American College of Chest Physicians/Society of Critical Care Medicine. Chest 1992, 101:1644-1655.

22. Lowenkron SE, Niederman MS: Definition and evaluation of the resolution of nosocomial pneumonia. Semin Respir Infect 1992, 7:271-281.

23. Nathens $A B$, Rotstein OD: Therapeutic options in peritonitis. Surg Clin North Am 1994, 74:677-692.

24. Wichmann MW, Inthorn D, Andress HJ, Schildberg FW: Incidence and mortality of severe sepsis in surgical intensive care patients: the influence of patient gender on disease process and outcome. Intensive Care Med 2000, 26:167-172.

25. Moreno R, Vincent JL, Matos R, Mendonca A, Cantraine F, Thijs L, Takala J, Sprung C, Antonelli M, Bruining $\mathrm{H}$, et al:: The use of maximum SOFA score to quantify organ dysfunction/failure in intensive care. Results of a prospective, multicentre study. Working Group on Sepsis related Problems of the ESICM. Intensive Care Med 1999, 25:686-696.

26. Kay $\mathrm{R}$, Little $\mathrm{S}$ : Transformation of the explanatory variables in the logistic regression model for binary data. Biometrika 1987, 74:495-501.

27. Hosmer DW, Lemeshow S: Applied Logistic Regression New York: Wiley; 1989:84.

28. Hosmer DW, Lemeshow S: Applied Survival Analysis New York: Wiley; 1999:158.

29. Therneau TM, Grambsch PM: Modeling Survival Data: Extending the Cox Mode/ Berlin, Heidelberg: Springer-Verlag; 2000:127.

30. Statistisches Bundesamt Wiesbaden, Germany [http:// www.destatis.de/download/d/bevoe/sterbet04ow.xls]

31. Ryan TA, Rady MY, Bashour CA, Leventhal M, Lytle B, Starr NJ: Predictors of outcome in cardiac surgical patients with prolonged intensive care stay. Chest 1997, 112:1035-1042.

32. Hughes M, MacKirdy FN, Norrie J, Grant IS: Outcome of longstay intensive care patients. Intensive Care Med 2001, 27:779-782.

33. Pajonk FG, Fischer A, Waydhas C, Bregenzer T, Schweiberer L: Outcome of long-term intensive therapy of surgery patients [in German]. Unfallchirurg 2002, 105:423-430.

34. Szalados JE: Age and functional status as determinants of intensive care unit outcome: sound basis for health policy or tip of the outcomes iceberg. Crit Care Med 2004, 32:291-293.

35. Kaplan V, Angus DC: Surviving intensive care. Crit Care Med 2002, 30:703-705

36. Angus DC, Carlet J: Surviving intensive care: a report from the 2002 Brussels Roundtable. Intensive Care Med 2003, 29:368-377.

37. Combes A, Costa MA, Trouillet JL, Baudot J, Mokhtari M, Gibert C, Chastre J: Morbidity, mortality, and quality-of-life outcomes of patients requiring $>$ or $=14$ days of mechanical ventilation. Crit Care Med 2003, 31:1373-1381.
38. Pappalardo F, Franco A, Landoni G, Cardano P, Zangrillo A, Alfieri $\mathrm{O}$ : Long-term outcome and quality of life of patients requiring prolonged mechanical ventilation after cardiac surgery. Eur J Cardiothorac Surg 2004, 25:548-552.

39. Staudinger T, Stoiser B, Mullner M, Locker GJ, Laczika K, Knapp S, Burgmann H, Wilfing Kofler J, Thalhammer F, Frass M: Outcome and prognostic factors in critically ill cancer patients admitted to the intensive care unit. Crit Care Med 2000, 28:1322-1328.

40. Azoulay E, Afessa B: The intensive care support of patients with malignancy: do everything that can be done. Intensive Care Med 2006, 32:3-5.

41. Bashour CA, Yared JP, Ryan TA: Long-term survival and functional capacity in cardiac surgery patients after prolonged intensive care. Crit Care Med 2000, 28:3847-3853.

42. Garland A: Improving the ICU: part 2. Chest 2005, 127:2165-2179.

43. Kelly WF, Eliasson AH, Stocker DJ, Hnatiuk OW: Do specialists differ on do-not-resuscitate decisions? Chest 2002, 121:957-963. 